OPEN

SUBJECT AREAS:

PHYSICS

MATERIALS SCIENCE

APPLIED PHYSICS

SYNTHESIS

Received

11 June 2012

Accepted

29 October 2012

Published

14 November 2012

Correspondence and requests for materials should be addressed to

J.K. (jingkong@mit. edu) or S.M.J.

(sungmij@mit.edu)

\section{A facile route for 3D aerogels from nanostructured ID and 2D materials}

\author{
Sung Mi Jung ${ }^{1}$, Hyun Young Jung ${ }^{2}$, Mildred S. Dresselhaus ${ }^{1,3}$, Yung Joon Jung ${ }^{2} \&$ Jing Kong ${ }^{1}$
}

'Department of Electrical Engineering and Computer Science, Massachusetts Institute of Technology, Cambridge, Massachusetts 02139, USA, '2Department of Mechanical and Industrial Engineering, Northeastern University, Boston, MA 021 15, USA,

${ }^{3}$ Department of Physics, Massachusetts Institute of Technology, Cambridge, Massachusetts 02139, USA.

Aerogels have numerous applications due to their high surface area and low densities. However, creating aerogels from a large variety of materials has remained an outstanding challenge. Here, we report a new methodology to enable aerogel production with a wide range of materials. The method is based on the assembly of anisotropic nano-objects (one-dimensional (1D) nanotubes, nanowires, or two-dimensional (2D) nanosheets) into a cross-linking network from their colloidal suspensions at the transition from the semi-dilute to the isotropic concentrated regime. The resultant aerogels have highly porous and ultrafine three-dimensional (3D) networks consisting of $1 \mathrm{D}\left(\mathrm{Ag}, \mathrm{Si}, \mathrm{MnO}_{2}\right.$, single-walled carbon nanotubes (SWNTs)) and 2D materials $\left(\mathrm{MoS}_{2}\right.$, graphene, h-BN) with high surface areas, low densities, and high electrical conductivities. This method opens up a facile route for aerogel production with a wide variety of materials and tremendous opportunities for bio-scaffold, energy storage, thermoelectric, catalysis, and hydrogen storage applications.

A erogels are extremely porous materials with large pore volumes and low bulk densities. Their unique structure imparts extraordinary properties and wide applications ${ }^{1-8}$. Despite the considerable need for aerogels, at present there are only limited types of materials that can be made into an "aerogel" structure. These include metal oxide aerogels (such as $\left.\mathrm{SiO}_{2}, \mathrm{Al}_{2} \mathrm{O}_{3}\right)^{9,10}$, carbon material aerogels (such as carbon, carbon nanotubes, graphene) ${ }^{11-14}$, semiconducting chalcogenide aerogels (such as $\left.\mathrm{CdS}, \mathrm{CdSe}, \mathrm{PbTe}\right)^{15,16}$, and more recently polysaccharide ${ }^{17}$ or protein-based aerogels ${ }^{18}$. Most aerogels are formed by extracting the liquid of a gel via critical point drying (CPD) in order that the gel network remains intact. The gel networks are assembled through a sol-gel process, where nanoparticle suspensions are formed and then the nanoparticles are crosslinked into 3D network branches by a suitable gelling agent precursor (Fig. S1a). Because of these specific cross-linking reactions, the material types of aerogels have been very limited. Therefore, there are a large number of interesting possibilities in creating a wide range of aerogels if gels can be assembled by the crosslinkng of the material itself without gel precursors.

We have recently come up with a new methodology to make gels, which will enable the synthesis of aerogel materials over a much wider range. This idea is inspired by the observation of gel formation. Gels consist of a solid 3D network that spans the volume of a liquid medium, and due to the surface tension by the solid structure, the liquid does not flow. If long chain molecules, or colloidal particles of 1D (i.e., nanowires or nanotubes) are dispersed in a liquid (e.g., water), when their concentrations in the water reaches a certain level, the chances for them to interlink with each other will be high, thus the flow of water is stopped and a gel forms. As a result, these $1 \mathrm{D}$ objects become the branches in the 3D network themselves (Fig. S1b). If the colloidal particles are 2D nanosheets, compared to a spherical particle of the same volume, these 2D nanosheets contribute a lot more to viscosity in their stretched state and the interlinking network from these $2 \mathrm{D}$ nanosheets will also form a gel (Fig. S1c). Therefore, in principle, a gel can be facilely made from almost any material by starting with the colloid suspensions of $1 \mathrm{D}$ nanowires/nanotubes or 2D nanosheets of various kinds of materials. And once a gel is formed, it is straightforward to develop an aerogel material by extracting the liquid via CPD.

\section{Results}

Formations of 1D nanowires, nanotubes, and 2D nanosheets aerogels. This methodology for various material aerogels production constitutes three steps: (i) the preparation of a well-dispersed, individual nanotube/ nanowire/nanosheet suspension; (ii) gel formation from dilute suspensions through a controlled concentration increase, and (iii) the supercritical drying of the gel to form an aerogel. Figure 1a is a schematic representation of the aerogel production. Here nanowires are shown as an example. A suspension of 

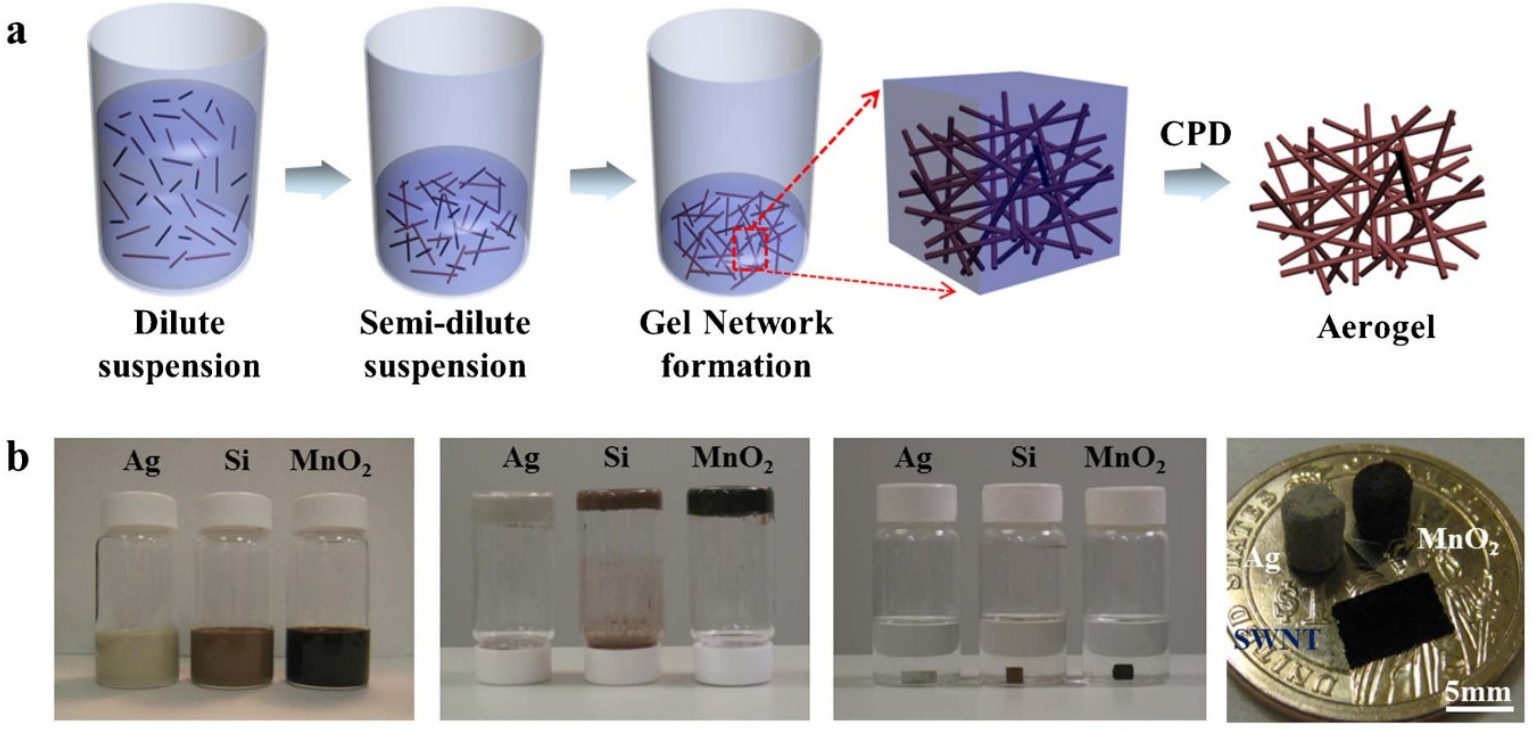

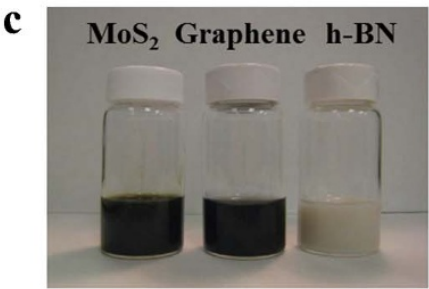

Dilute suspensions

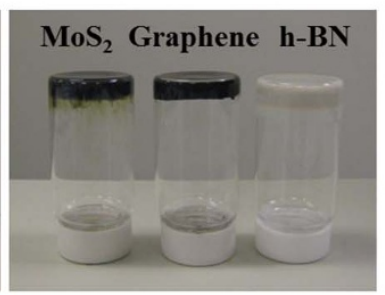

Gel formation

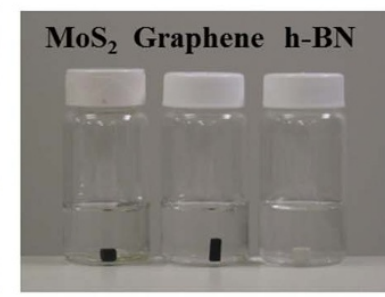

Surfactant-removed

gels

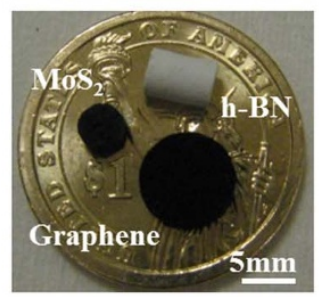

Aerogel

Figure 1 | The gel/aerogel production process (illustration (a) and actual process (b, c)). a, Schematic representation of nanowire/nanotube aerogel production via method developed in this work. $\mathrm{b}$, Photographs of dilute suspensions, gels, and surfactant-removed gels of $\mathrm{Ag}, \mathrm{Si}$, and $\mathrm{MnO}_{2}$ nanowires, and aerogels of $\mathrm{Ag}, \mathrm{MnO}_{2}$ and SWNT. c, Photographs of dilute suspensions, gels, surfactant-removed gels, and aerogels of $\mathrm{MoS}_{2}$, graphene, and h-BN nanosheet.

nanowires is initially uniformly dispersed with/without surfactant in ethanol using ultra-sonication at a low concentration, and then the dilute suspension transforms into a more concentrated state to form a gel network by evaporation of the solvent at low temperature $(313 \mathrm{~K})$ with a large volume compression. At higher concentration the suspension becomes a gel consisting of a 3D network of nanowires. The resultant gel is then set into various shapes using molds and the molded gel is transferred into water. The water is maintained at $353 \mathrm{~K}$ and changed several times to remove the surfactants. After that, the gels are supercritically dried into aerogels to retain the original gel volume (see Supplementary Information for a more detailed method). There are two key elements in this method: first is the homogeneous dispersion at dilute concentration, which prevents nanowire/nanotube/nanosheet bundling or agglomeration; second is the slow evaporation process of the solvent, which helps to reduce the volume of the nanowire/nanotube/nanosheet suspension to produce a concentrated suspension without nanowire/nanotube/nanosheet aggregation.

In this work, as shown in Fig. $\mathrm{lb}$ and $\mathrm{c}, \mathrm{Ag}, \mathrm{Si}, \mathrm{MnO}_{2}, \mathrm{SWNT}$, $\mathrm{MoS}_{2}$, graphene, and h-BN were selected because of a variety of potential applications. The Ag nanowire aerogel is a great candidate for a scaffold for tissue engineering, and Si nanowires can be used as thermoelectric materials. $\mathrm{MnO}_{2}$ nanowire, SWNT, and graphene aerogels have a huge potential for supercapacitors and batteries. In addition, $\mathrm{MoS}_{2}$ and h-BN aerogels can be useful for catalysts and $\mathrm{H}_{2}$ storage, respectively. The second photograph in Fig. $1 \mathrm{~b}$ shows the vials containing the suspensions which are turned up-side down, confirming the gel formation from the more concentrated suspension of nanowires/nanotubes. The shape of the gels could be readily manipulated by different molds so that aerogels have various desired shapes. Figure 1c shows photographs of the gels and aerogels and the formation processes of gels with $2 \mathrm{D}$ materials, and those illustrated here are $\mathrm{MoS}_{2}$, graphene, and h-BN gel/aerogels.

Gel formation concentration. Based on the Doi and Edwards theory which describes the phase behavior of a suspension of rod-like molecules $^{19}$, the range of gel formation concentrations can be predicted in this work. Here the term "nanowire" is used to represent both nanowires and nanotubes. The dynamic behavior of nanowires in a suspension depends on the concentration and the aspect ratio of the nanowires. Thus, based on the degree of particle interaction and the excluded volume of a nanowire, the nanowire in a suspension can be classified into four concentration regimes as: dilute solution, semidilute solution, isotropic concentrated solution, and liquid crystalline (Fig. S2). Following this theory, we consider a suspension of $n$ nanowires per unit volume, each nanowire with a length $L$ and a diameter $d$ and a corresponding aspect ratio $a_{r}=L / d$. The nanowire concentration described through the volume fraction $\varphi$ is defined ${ }^{20}$ as

$$
\varphi=\frac{\pi n L d^{2}}{4}=\frac{\pi n L^{3}}{4 a_{r}^{2}}
$$

As the concentration of the nanowire suspension increases, the rotational motion of each nanowire is inhibited by other nanowires and some physical contact interaction between nanowires is present $^{21}$. At a still higher concentration, the nanowire suspension forms a 3D continuous nanowires network due to physical bond formation at the contact point between the nanowires by van der Waals forces. The gel formation occurs at the transition from the 
Table 1 | The theoretical $(\varphi$ gel $)$ and experimental gel formation concentration $\left(\varphi^{\prime}\right.$ gell of nanowires

\begin{tabular}{lccccl}
$\mathrm{NWs}$ & $d_{m}(n m)$ & $L_{m}(\mu m)$ & $a_{r}$ & $\varphi$ gel & $\varphi^{\prime}{ }_{\text {gel }}$ \\
\hline $\mathrm{Ag}$ & 113 & 13.7 & 121 & 0.0083 & 0.0071 \\
$\mathrm{Si}$ & 41 & 5.2 & 127 & 0.0079 & 0.007 \\
$\mathrm{MnO}_{2}$ & 19 & 8.4 & 442 & 0.0023 & 0.0018
\end{tabular}

Average diameter $\left(d_{m}\right)$, average length $\left(L_{m}\right)$, average aspect ratio $\left(a_{r}\right)$, theoretical gel formation concentration $\left(\varphi_{\text {gel }}\right)$, average experimental gel formation concentration $\left(\varphi^{\prime}\right.$ gel $)$ of $\mathrm{Ag}, \mathrm{Si}$, and $\mathrm{MnO}_{2}$ nanowires forming gels and aerogels. The diameter and length of nanowires were measured by atomic force microscope (AFM), scanning electron microscope (SEM), respectively.

semi-dilute to isotropic concentrated regime $\mathrm{e}^{22-24}$. The gel formation concentration is $\varphi=a_{r}{ }^{-1}$ when $n=1 /\left(d L^{2}\right)$. Thus we define this concentration to be the theoretical gel formation concentration $\varphi_{\text {gel }}$, and $\varphi_{\text {gel }}=a_{r}^{-1}$.

Our experimental observation is consistent with this theoretical gel formation concentration $\left(\varphi_{\text {gel }}\right)$ based upon the Doi and Edwards theory. The average aspect ratio values of $\mathrm{Ag}, \mathrm{Si}$, and $\mathrm{MnO}_{2}$ nanowires were obtained as 121,127 , and 442 , respectively, from their average diameter and length (see Table 1 and Fig. S3). Thus the theoretical gel formation concentration $\varphi$ gel of $\mathrm{Ag}, \mathrm{Si}$, and $\mathrm{MnO}_{2}$ nanowires should be $0.0083,0.0079$, and 0.0023 , respectively, and the average experimental gel transition concentrations $\left(\varphi_{g e l}^{\prime}\right)$ of $\mathrm{Ag}, \mathrm{Si}$, and $\mathrm{MnO}_{2}$ nanowires were found to be $0.0071,0.007$, and 0.0018 , respectively. As a result, we could confirm that the $\varphi_{g e l}^{\prime}$ values are very close to the $\varphi_{\text {gel }}$ value and indeed the gels are formed near the $\varphi_{\text {gel }}$.

In the case of $2 \mathrm{D}$ materials, the dilute suspensions were concentrated and transformed into the gels of 2D sheets at 3.2, 1, and 2.1 wt $\%$ for $\mathrm{MoS}_{2}$, graphene, and h-BN nanosheets, respectively. The relation between the sheet aspect ratio and the gel formation concentration shall be further considered in future work.

Structures of the aerogels. The SEM images of $\mathrm{Ag}, \mathrm{MnO}_{2}$, $\mathrm{Si}$, and SWNT aerogels in Fig. 2a, b, and Fig. S4a, b displays 3D networks with ultrafine $1 \mathrm{D}$ nanowire/nanotube structures. The $2 \mathrm{D}$ material aerogels show a porous $3 \mathrm{D}$ network of randomly oriented sheet-like structures (Fig. 2c and Fig. S5). These images indicate highly porous nanowire/nanotube/nanosheet networks with pore sizes in the range of a few hundred nanometers to a few micrometers. The key factors of the formation of an unbundled or non-aggregated nanowire/ nanotube/nanosheet network are the homogeneous dispersion of the dilute suspensions and the slow evaporation of the solvent. In the case of nanowires with small diameters, they were dispersed at low concentrations with surfactants to prevent the strong van der Waals attraction between small diameter nanowires. The uniformly dispersed dilute suspension was then slowly compressed up to the gel formation concentration by evaporating the solvent at low temperature to avoid nanowire aggregation. On the other hand, nanowire gels formed from non-uniformly dispersed suspensions without surfactant or directly from a concentrated suspension produce bundled nanowire networks (compare Fig. S6a with S6b).

\section{Discussion}

The porosities of $\mathrm{Ag}, \mathrm{Si}, \mathrm{MnO}_{2}$, SWNT, and graphene aerogels were confirmed by $\mathrm{N}_{2}$ adsorption-desorption analysis. Figure $3 \mathrm{a}$ and $3 \mathrm{~b}$ show the $\mathrm{N}_{2}$ adsorption-desorption isotherm and pore size distribution of $\mathrm{MnO}_{2}$ nanowire aerogel as an example. All the aerogels showed a type IV isotherm with H3-type hysteresis loop, which indicate that mesopores have cylindrical pore geometries (Fig. 3a and Fig. S7) ${ }^{25}$. Their surface areas were calculated with the Brunauer-Ennett-Teller (BET) method (Table 2). The ultra-fine porous nanowire networks result in high surface areas. The $\mathrm{MnO}_{2}$ nanowire aerogel has a surface area of $82.2 \mathrm{~m}^{2} \mathrm{~g}^{-1}$ (Fig. 3a, which is 2 times larger than the surface area of $\mathrm{MnO}_{2}$ nanowire membrane with the same starting $\mathrm{MnO}_{2}$ nanowire material ${ }^{26}$. The surface area value of
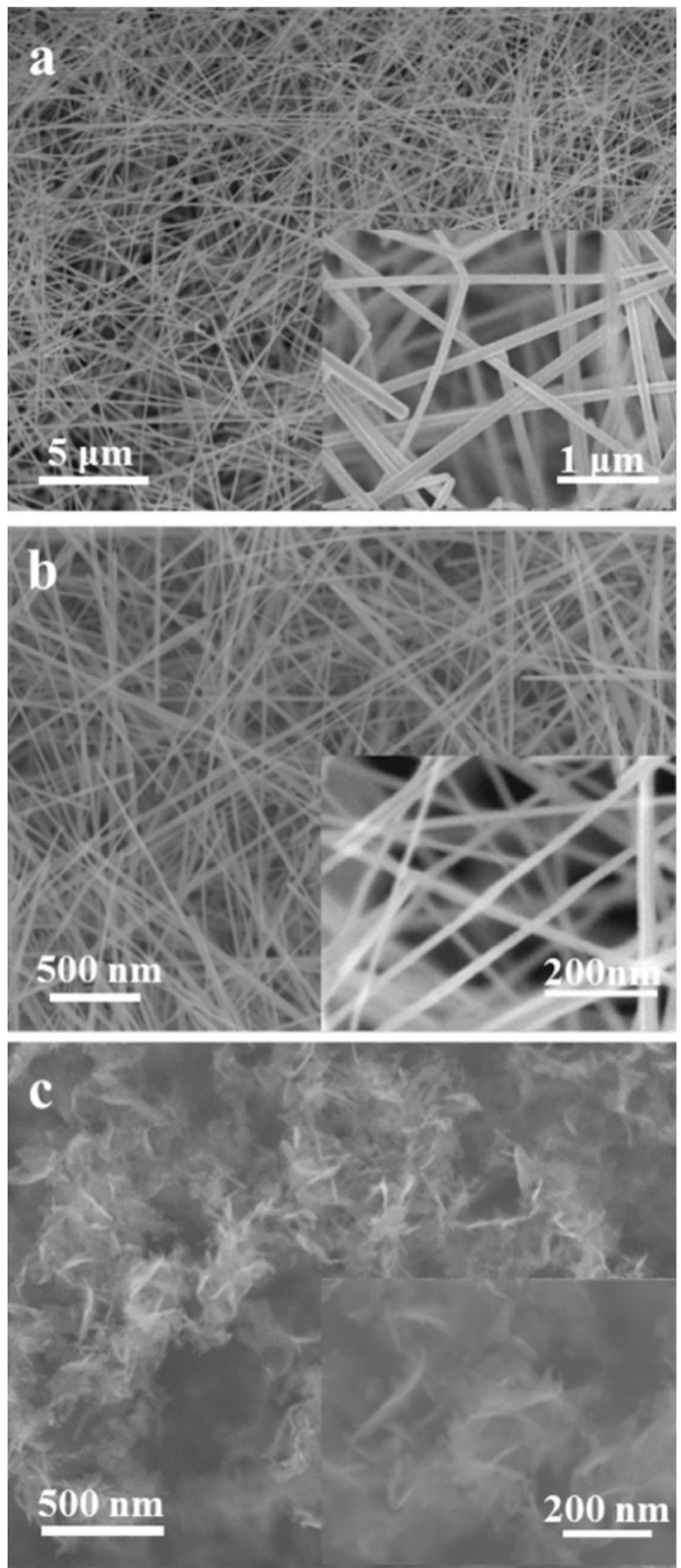

Figure $2 \mid$ The 3D network structures of $\mathrm{Ag}$ nanowire, $\mathrm{MnO}_{2}$ nanowire, and graphene aerogels. Low magnification SEM images of Ag nanowires (a), $\mathrm{MnO}_{2}$ nanowires (b), and graphene nanosheets (c) networks. The insets show a higher magnification of the aerogels. a and b consist of highly porous and ultrafine nanowires networks with pore sizes in the range of a few hundred nanometers to a few micrometers. c shows a porous $3 \mathrm{D}$ network of randomly oriented sheet-like structures.

$1011 \mathrm{~m}^{2} \mathrm{~g}^{-1}$ for a SWNT aerogel is 5.5 times higher than the best reported SWNT-based aerogels ${ }^{27}$ and also close to the theoretically calculated specific surface area $\left(1315 \mathrm{~m}^{2} \mathrm{~g}^{-1}\right.$ for SWNTs $\left.{ }^{28}\right)$. The aerogels have also low densities, as shown in Table 2. The Ag nanowire aerogel density is $88 \mathrm{mg} / \mathrm{cm}^{3}$, relatively high due to the large diameter (average diameter is $113 \mathrm{~nm}$ ), but a SWNT aerogel has the 

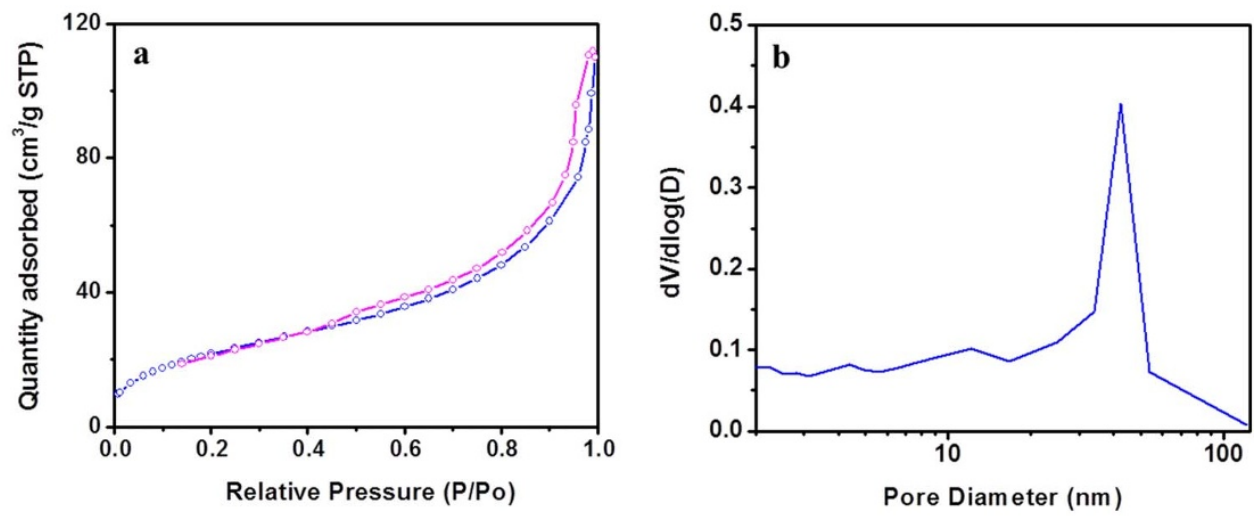

Figure 3 Porosity of $\mathrm{MnO}_{2}$ nanowire aerogel by $\mathrm{N}_{2}$ adsorption-desorption analysis. a, $\mathrm{N}_{2}$ adsorption/desorption isotherm at $77 \mathrm{~K}$ (solid circle, adsorption data; open circle, desorption data). b, Pore size distribution as determined by the Barrett-Joynes-Halenda (BJH) method, shows the presence of a range of pores extending from the meso to the macro regime, with a peak pore diameter of $43 \mathrm{~nm}$.

lowest density of $2.7 \mathrm{mg} / \mathrm{cm}^{3}$, compared with those of previously reported the SWNT or multi-walled nanotube (MWNT) aerogels $^{27,29,30}$ and only two times higher than an air density of $1.2 \mathrm{mg} /$ $\mathrm{cm}^{3}$.

As can be seen from the SEM images of the aerogels produced in this work, the branches of the 3D networks consist of highly crystalline nanowires or nanosheet material. In contrast, aerogels produced by conventional methods typically have interlinking nanocrystallites (as illustrated by Fig. S1a vs. Fig. S1b, c). As a result, it can be anticipated that the aerogels produced by this work should have good electrical conductivities, as the electrical conduction in the conventional aerogels normally occur via electron hopping between the nanocrystallites (Fig. S1a), whereas in the aerogels developed by this work the nanowires or nanosheets directly provide the path for electrical conduction. Indeed, the electrical conductivity of an Ag nanowire aerogel is $3 \times 10^{6} \mathrm{~S} / \mathrm{m}$ for an aerogel density of $88 \mathrm{mg} / \mathrm{cm}^{3}$ (which is the gel formation concentration) (Table 2). This value is comparable to the conductivity of thick $(600 \mathrm{~nm}) \mathrm{Ag}$ nanowires films ${ }^{31}$, and on the same order of magnitude as bulk silver $(9.4 \times$ $\left.10^{6} \mathrm{~S} / \mathrm{m}\right)^{32}$. For other aerogels, we obtained $91 \mathrm{~S} / \mathrm{m}$ for SWNT and $273 \mathrm{~S} / \mathrm{m}$ for graphene aerogels at densities corresponding to their gel formation concentrations $\left(2.7 \mathrm{mg} / \mathrm{cm}^{3}\right.$ and $13 \mathrm{mg} / \mathrm{cm}^{3}$, respectively). When aerogels are made at higher densities, even a higher electrical conductivity can be achieved. For example, $302 \mathrm{~S} / \mathrm{m}$ was obtained for a SWNT aerogel at $20 \mathrm{mg} / \mathrm{cm}^{3}$, while a graphene aerogel with $24 \mathrm{mg} / \mathrm{cm}^{3}$ shows a conductivity of $390 \mathrm{~S} / \mathrm{m}$. These values are 3 $\sim 5$ times higher than values previously reported ${ }^{13,27}$. Similarly, it is anticipated that these aerogels should have different mechanical properties compared to the conventional aerogels. The conventional aerogels (as shown in Fig. S1a) have low tensile strength and elastic modulus (i.e. they are brittle and will readily fragment when bent) because in these aerogels, the branches of the 3D network are formed by many nanocrystallites interlinked together, which tends to break

Table 2 | BET surface areas, densities, and electrical conductivities for $\mathrm{Ag}, \mathrm{Si}, \mathrm{MnO}_{2}, \mathrm{SWNT}$, and graphene aerogels made at gel formation concentration $\varphi^{\prime}$ gel

\begin{tabular}{lccc|} 
Aerogel & $\begin{array}{c}\text { Surface } \\
\text { area }\left(\mathrm{m}^{2} / \mathrm{g}\right)\end{array}$ & $\begin{array}{c}\text { Density } \\
\left(\mathrm{mg} / \mathrm{cm}^{3}\right)\end{array}$ & $\begin{array}{c}\text { Electrical } \\
\text { conductivity } \\
(\mathrm{S} / \mathrm{m})\end{array}$ \\
\hline $\mathrm{Ag}$ & 5.5 & 88 & $3 \times 10^{6}$ \\
$\mathrm{Si}$ & 69.4 & 24.6 & $\mathrm{n} / \mathrm{a}$ \\
$\mathrm{MnO} 2$ & 82.2 & 12 & $\mathrm{n} / \mathrm{a}$ \\
$\mathrm{SWNT}$ & 1011 & 2.7 & 91 \\
Graphene & 605 & 13 & 273 \\
\hline
\end{tabular}

with applied forces ${ }^{14}$. In contrast, the nanotubes/nanowires in the aerogels in this work can easily accommodate elastic bending, thus these aerogels should be very elastic. Indeed super-elastic behavior has been observed for $\mathrm{MnO}_{2}$ nanowire aerogels, and further mechanical characterization is currently underway.

It should be noted that the properties of the aerogel materials developed in this work will rely on the starting nanowires/nanotubes/nanosheets materials. If the starting nanowires have long lengths and small diameters, an aerogel with a high surface area, low density, high electrical conductivity, and high tensile strength can be anticipated.

In conclusion, the strategy developed in this work to form gels can be applied to many materials by starting with the colloid suspension of either 1D nanowires/nanotubes or 2D nanosheets. Once a gel is formed, it is straightforward to develop an aerogel material by extracting the liquid via supercritical drying. This method provides a straightforward yet powerful and potentially general route for the creation of a wide variety of aerogels. In principle, aerogels from any type of materials can be enabled by this method. Combining their individual material properties (e.g., high electrical conductivity from a metal, or optical properties like photoluminescence of a semiconductor) with the advantages imparted by the structure (such as high surface area and low densities), it can be anticipated that not only much wider opportunities will be opened up for the typical applications of aerogels (such as catalysis, sensing, thermoelectrics, scaffolds for tissue engineering, supercapacitors, batteries and solar cells) but potentially novel applications as well.

\section{Methods}

Materials. Ag and Si nanowires were purchased from Blue Nano (SLV-NW-90) and Aldrich (product number: 731498), respectively. $\mathrm{MnO}_{2}$ nanowires were synthesized by hydrothermal method. The starting materials were composed of $19.1 \mathrm{mmol}$ of potassium sulphate $\left(\mathrm{K}_{2} \mathrm{SO}_{4}\right)$, potassium persulphate $\left(\mathrm{K}_{2} \mathrm{~S}_{2} \mathrm{O}_{8}\right)$ and manganese sulphate monohydrate $\left(\mathrm{MnSO}_{4} \cdot \mathrm{H}_{2} \mathrm{O}\right)$ in a ratio of 1:2:1 in $80 \mathrm{ml}$ deionized (DI) water $^{26}$. The suspension was transferred to a Teflon vessel and the sealed vessel was heated at in an oven at $523 \mathrm{~K}$ for 4 days ${ }^{26}$. The synthesized $\mathrm{MnO}_{2}$ nanowires were suspended in $800 \mathrm{ml}$ of DI water. The suspension was filtered and washed several times until all soluble impurities were removed from the solid and then the nanowires were dried in a vacuum oven at $353 \mathrm{~K}$ for $24 \mathrm{hr}$.

Purified HIPCO Single-Wall Carbon Nanotubes (SWNTs) were obtained from Unidym, Inc and $\mathrm{MoS}_{2}$, graphite, and h-BN powders were obtained from Aldrich (CAS number: 1317-33-5), Alfa Asear (product number: 43319), Aldrich (CAS number: 10043-11-5), respectively. Linear sodium dodecylbenzene sulfonate (SDBS) and planar sodium cholate (SC) were used as surfactant for dispersion of $1 \mathrm{D}$ and $2 \mathrm{D}$ materials, respectively.

Formations of 1D nanowires, nanotubes, and 2D nanosheets gels. Firstly, Ag and Si nanowires were each dispersed via ultra-sonication (Branson 2510 sonicator) in ethanol without, and with SDBS for $10 \sim 60 \mathrm{~min}$, respectively. The $\mathrm{MnO}_{2}$ nanowires were sonicated in ethanol with SDBS for $2 \mathrm{~h}$. The concentrations of $\mathrm{Ag}, \mathrm{Si}$, and $\mathrm{MnO}_{2}$ 
nanowires were 10,3 , and $2 \mathrm{mg} \cdot \mathrm{mL}^{-1}$, respectively. The surfactant/nanowires weight ratios were all 5:1.

In the case of SWNT gels, the suspension of $0.1 \mathrm{wt} \%$ SWNTs was dispersed in DI water with SDBS at a SWNT: SDBS ratio of 1:5 for $5 \sim 15$ h by Branson 2510 sonicator. The $\mathrm{MoS}_{2}$, graphite, and h-BN powders were sonicated in $2 \mathrm{wt} \% \mathrm{SC}$ solution for $4 \sim 15 \mathrm{~h}$. The concentrations of $\mathrm{MoS}_{2}$, graphite, and h-BN powders were 66,85 , and $66 \mathrm{mg} \cdot \mathrm{mL}^{-1}$, respectively ${ }^{33,34}$. The SWNT suspension was centrifuged (Eppendorf centrifuge $5804 \mathrm{R}$ ) to separate bundles from the individual nanotubes at $11 \mathrm{k} \mathrm{rpm}$ for $1 \sim 2 \mathrm{~h}$. The exfoliated $\mathrm{MoS}_{2}$, graphene, and h-BN sheets were centrifuged at $1500 \mathrm{rpm}$ for $1 \mathrm{~h}$ to remove the non-exfoliated powders. The supernatant in each case was collected after centrifugation and measured with a Cary 6000i UVVis-NIR spectrophotometer. The concentration of the $\mathrm{MoS}_{2}$, graphene, SWNT, and h-BN suspensions were calculated using an absorption coefficient $(\alpha)$ of 1517, 2460, and 2600 absorbance $\mathrm{mL} \cdot \mathrm{mg}^{-1} \mathrm{~m}^{-1}$ at a wavelength of 672,660 , and $930 \mathrm{~nm}$, respectively ${ }^{33-35}$.

The dilute suspensions of $\mathrm{Ag}, \mathrm{Si}, \mathrm{MnO}_{2}, \mathrm{SWNT}, \mathrm{MoS}_{2}$, graphene, and h-BN were evaporated at $313 \mathrm{~K}$ to transform them into more concentrated suspensions (gels) with a large volume compression. The resultant gels were set into a cylinder or rectangular shaped molds for several hours and the molded gels were transferred to DI water. The solutions were heated up $353 \mathrm{~K}$ to remove surfactant and changed at least 4 to 5 times over several days. This step removed surfactant, as shown in the SEM images (Fig. 2, Fig. S4, and Fig. S5).

The Ag, Si, $\mathrm{MnO}_{2}, \mathrm{SWNT}, \mathrm{MoS}_{2}$, graphene, and h-BN gels were placed into anhydrous ethanol overnight for solvent exchange and then dried at 1,200 psi and $308 \mathrm{~K}$ by using a critical point drier (CPD, 13200J-AB, SPI supplies) into the Ag, $\mathrm{Si}$ $\mathrm{MnO}_{2}, \mathrm{SWNT}, \mathrm{MoS}_{2}$, graphene, and h-BN aerogels keeping the gel network intact (Fig. 2, Fig. S4, and Fig. S5).

Characterization. Atomic force microscope (AFM) measurements were carried out to characterize the diameter distribution of nanowires with a Dimension 3100, Veeco system. Scanning electron microscopy (JEOL 6700F) was used to find the length distribution of nanowires and the porosities of the aerogel networks. Nitrogen adsorption and desorption isotherms for the porosities of the aerogels were measured at $77 \mathrm{~K}$ on a Micromeritics ASAP 2020 system. Before measurement, the samples were degassed at $423 \mathrm{~K}$ under vacuum $\left(<10^{-4} \mathrm{mbar}\right)$ for several hours. Surface areas of the aerogels were calculated from the Brunauer-Ennett-Teller (BET) method multimolecular layer adsorption model. The electrical conductivities of the Ag nanowire, SWNT, and graphene aerogels at room temperature were measured using a four-probe station for porous materials ${ }^{36}$. The resulting errors in the electrical conductivity measurement are estimated to be less than $5 \%$.

1. Olsson, R. T. Making flexible magnetic aerogels and stiff magnetic nanopaper using cellulose nanofibrils as templates. Nat. Nanotechnol. 5, 584-588 (2010).

2. Aliev, A. E. et al. Giant-stroke, superelastic carbon nanotube aerogel muscles. Science 323, 1575-1578 (2009).

3. Wei, T. Y. et al. A cost-effective supercapacitor material of ultrahigh specific capacitances: spinel nickel cobaltite aerogels from an epoxide-driven sol-gel process. Adv. Mater. 22, 347-351 (2010).

4. Hamann, T. W., Martinson, A. B., Elam, F. J. W., Pellin, M. J. \& Hupp, J. T. Aerogel templated $\mathrm{ZnO}$ dye-sensitized solar cells. Adv. Mater. 20, 1560-1564 (2008).

5. Korhonen, J. T. et al. Inorganic hollow nanotube aerogels by atomic layer deposition onto native nanocellulose templates. ACS Nano 22, 1967-1974 (2011).

6. Yao, Q. \& Brock, S. L. Optical sensing of triethylamine using CdSe aerogels. Nanotechnology 21, 115502/1-115502/10 (2010).

7. Li, G. et al. Mesoporous $\mathrm{MnO}_{2} /$ carbon aerogel composites as promising electrode materials for high-performance supercapacitors. Langmuir 26, 2209-2213 (2010).

8. Jin, H. at al. Superhydrophobic and superoleophobic nanocellulose aerogel membranes as bioinspired cargo carriers on water and oil. Langmuir 27, 1930-1934 (2011).

9. Moner-Girona, M., Roig, A., Molins, E. \& Llibre, J. Sol-gel route to direct formation of silica aerogel microparticles using supercritical solvents. J. Sol-Gel Sci. Tech. 26, 645-649 (2003).

10. Baumann, T. F. et al. Synthesis of high-surface-area alumina aerogels without the use of alkoxide precursors. Chem. Mater. 17, 395-401 (2005).

11. Horikawa, T., Hayashi, J. \& Muroyama, K. Size control and characterization of spherical carbon aerogel particles from resorcinol-formaldehyde resin. Carbon 42, 169-175 (2004).

12. Worsley, A. et al. Synthesis and characterization of monolithic carbon aerogel nanocomposites containing double-walled carbon nanotubes. Langmuir 24, 9763-9766 (2008).

13. Marcus, A. W. et al. Synthesis of Graphene Aerogel with High Electrical Conductivity. J. Am. Chem. Soc. 132, 14067-14069 (2010).

14. Aegerter, M. A., Leventis, N. \& Koebel, M. M. Aerogels Handbook (SpringerVerlag New York, 2011).
15. Mohanan, J. L., Arachchige, I. U. \& Brock, S. L. Porous Semiconductor Chalcogenide Aerogels. Science 307, 397-400 (2005).

16. Santanu, B., Pantelis, N. T., Peter, J. C., Gerasimos, S. A. \& Mercouri, G. K. Porous Semiconducting Gels and Aerogels from Chalcogenide Clusters. Science 317, 490-493 (2007).

17. García-González, C. A., Alnaief, M. \& Smirnova. I. Polysaccharide-based aerogels-Promising biodegradable carriers for drug delivery systems. Carbohydr. Polym. 86, 1425-1438 (2011).

18. Betz, M., García-González, C. A., Subrahmanyamb, R. P., Smirnova, I. \& Kulozik. U. Preparation of novel whey protein-based aerogels as drug carriers for life science applications. J. of Supercritical Fluids 72, 111-119 (2012).

19. Doi, M. \& Edward, S. F. The Theory of Polymer Dynamics (Oxford University Press, 1986).

20. Eberle, A. P. R., Baird, D. G. \& Wapperom, P. Rheology of non-newtonian fluids containing glass fibers: a review of experimental literature. Ind. Eng. Chem. Res. 47, 3470-3488 (2008).

21. Davis, V. A. Liquid crystalline assembly of nanocylinders. J. Mater. Res. 26, 140-153 (2011).

22. Balberg, I., Binenbaum, N. \& Wagner, N. Percolation thresholds in the threedimensional sticks system. Phys. Rev. Lett. 52, 1465-1468 (1984).

23. Balberg, I., Anderson, C. H., Alexander, S. \& Wagner, N. Excluded volume and its relation to the onset of percolation. Phys. Rev. B 30, 3933-3943 (1984).

24. Bug, A. L. R., Safran, S. A. \& Webman, I. Continuum percolation of rods. Phys. Rev. Lett. 54, 1412-1415 (1985).

25. Gregg, S. J. \& Sing, K. S. W. Adsorption, Surface Area and Porosity (Academic Press, New York, 1982).

26. Yuan, J. et al. Superwetting nanowire membranes for selective absorption. Nat. Nanotechnol. 3, 332-336 (2008).

27. Worsley, M. A. et al. Properties of single-walled carbon nanotube-based aerogels as a function of nanotube loading. Acta Materialia 57, 5131-5136 (2009).

28. Peigney, A., Laurent, C., Flahaut, E., Bacsa, R. R. \& Rousset, A. Specific surface area of carbon nanotubes and bundles of carbon nanotubes. Carbon 39, 507-514 (2001).

29. Bryning, M. B. et al. Carbon nanotube aerogel. Adv. Mater. 19, 661-664 (2007)

30. Zou, J. et al. Ultralight multiwalled carbon nanotube aerogel. ACS Nano 4, 7293-7302 (2010).

31. De, S. et al. Silver nanowire networks as flexible, transparent, conducting films: extremely high DC to optical conductivity ratios. ACS Nano 3, 1767-1774 (2009)

32. de Vries, A. J. et al. Ellipsometric study of percolation in electroless deposited silver films. J. Appl. Phys. 101, 053703/1-053703/10 (2007).

33. Smith, R. J. et al. Large-scale exfoliation of inorganic layered compounds in aqueous surfactant solutions. Adv. Mater. 23, 3944-3948 (2011).

34. Green, A. A. \& Hersam, M. C. Emerging methods for producing monodisperse graphene dispersions. J. Phys. Chem. Lett. 1, 544-549 (2010).

35. Fagan, J. A. et al. Length fractionation of carbon nanotubes using centrifugation. Adv. Mater 20, 1609-1613 (2008)

36. Dharmasena, K. P. \& Wadley, H. N. G. Electrical conductivity of open-cell metal foams. J. Mater. Res. 17, 625-631 (2002).

\section{Acknowledgments}

The authors acknowledge the funding from MIT Energy Initiative, National Science Foundation award number NSF DMR 0845358 and the generous gift from Doug Spreng. S. M. J. acknowledges MIT Institute of Soldier Nanotechnologies (ISN) for the access of various instruments to carry out this work and the help from Prof. Pablo Jarillo-Herrero, Britt Baugher, Dr. Mario Hofmann and Yongcheol Shin and Dr. Tingying (Helen) Zeng.

\section{Author Contributions:}

S.M.J. and J.K. designed experiments and S.M.J. carried out most experiments and H.Y.J. carried out electrical conductivity measurements. S.M.J., H.Y.J., and J.K. discussed the results. S.M.J. wrote the manuscript and all authors contributed to revisions.

\section{Additional information}

Supplementary Information: accompanies this paper at http://www.nature.com/ scientificreports.

Competing financial interests: The authors declare no competing financial interests.

License: This work is licensed under a Creative Commons

Attribution-NonCommercial-NoDerivative Works 3.0 Unported License. To view a copy of this license, visit http://creativecommons.org/licenses/by-nc-nd/3.0/.

How to cite this article: Jung, S.M., Jung, H.Y., Dresselhaus, M.S., Jung, Y.J. \& Kong, J. A facile route for 3D aerogels from nanostructured 1D and 2D materials. Sci. Rep. 2, 849, DOI:10.1038/srep00849 (2012) 
SUBJECT AREAS: PHYSICS

MATERIALS SCIENCE

APPLIED PHYSICS

SYNTHESIS

SCIENTIFIC REPORTS:

$1: 849$

DOI: $10.1038 /$ srep00849

(2012)

Published:

14 November 2012

Updated:

8 March 2013
CORRIGENDUM: A facile route for 3D aerogels from nanostructured 1D and 2D materials

Sung Mi Jung, Hyun Young Jung, Mildred S. Dresselhaus, Yung Joon Jung \& Jing Kong

The authors regret that previous work reporting the production of aerogels from nanotubes was not properly acknowledged and cited in our paper. These references appear below ${ }^{1-3}$. Reference 1 was referenced in the published paper (reference 29), but was only cited to compare the aerogel properties instead of acknowledging what has already been studied.

1. Bryning, M. B., Milkie, D. E., Islam, M. F., Hough, L. A., Kikkawa, J. M. \& Yodh, A. G. Carbon Nanotube Aerogels. Adv. Mater. 19, 661-664 (2007).

2. Hough, L. A., Islam, M. F., Janmey, P. A. \& Yodh, A. G. Viscoelasticity of Single Wall Carbon Nanotube Suspensions. Phys Rev Lett 93, 168102 (2004).

3. Hough, L. A., Islam, M. F., Hammouda, B., Yodh, A. G. \& Heiney, P. A. Structure of Semidilute Single-Wall Carbon Nanotube Suspensions and Gels. Nano Letters 6, 313 (2006). 